\title{
Grating formation in step flow heterogeneous growth and wavelength selection induced by confinement
}

\author{
F. Picaud ${ }^{\text {a }}$, C. Ramseyer ${ }^{\mathrm{a}, *}$, C. Girardet ${ }^{\mathrm{a}}$, H. Brune ${ }^{\mathrm{b}}$, K. Kern ${ }^{\mathrm{b}, \mathrm{c}}$ \\ a Laboratoire de Physique Moléculaire, UMR 6624, Faculté des Sciences, La Bouloie, Université de Franche-Comté, \\ F25030 Besançon Cedex, France \\ ${ }^{\mathrm{b}}$ Institut de Physique des Nanostructures, EPFL, CH-1015 Lausanne, Switzerland \\ c Max-Planck-Institut für Festkörperforschung, Heisenbergstr. 1 D-70569 Stuttgart, Germany
}

Received 25 August 2003; accepted for publication 26 January 2004

\begin{abstract}
Based on kinetic Monte Carlo simulations, we show that modulated wires and island gratings can be formed at vicinal surfaces. The modulation (grating) wavelength along the steps can be tailored by external conditions (coverage and temperature) and intrinsic surface properties (diffusion, terrace width) via a scaling law. Above $220 \mathrm{~K}$ a thermodynamic saturation value for the wavelength occurs, which depends only on the terrace width. This morphological behavior can be understood in terms of nucleation arguments applied to heteroepitaxial growth of $\mathrm{Ag}$ on stepped $\operatorname{Pt}\left(\begin{array}{lll}1 & 1 & 1) \\ ) & \text { surfaces. }\end{array}\right.$
\end{abstract}

(c) 2004 Elsevier B.V. All rights reserved.

Keywords: Monte Carlo simulations; Adsorption kinetics; Growth; Silver; Platinum; Surface defects

Growth of new materials with low dimensional behavior and tailored properties such as quantum dots, nanowires and nanogratings has become a major challenge. These structures, generally obtained using molecular beam epitaxy (MBE), result from a subtle balance between energetic (lateral and holding interaction energies) and kinetic (deposition, diffusion, aggregation, evaporation) processes [1-4]. Many efforts were done to identify and control the atomic processes respon-

\footnotetext{
${ }^{*}$ Corresponding author. Tel.: +33-3-81-66-64-82; fax: +33-381-66-64-75.

E-mail address: christophe.ramseyer@univ-fcomte.fr (C. Ramseyer).
}

sible for the nanostructure growth. The use of defects like steps or periodic strain patterns has proven to be a very promising way to achieve the desired nanostructuring [5-8].

The surface steps are known to play a crucial role in the growth. Schematically, from an energetic point of view, they act as sinks for the deposited atoms. The gain (resp. loss) of coordination at the lower (resp. upper) step edges leads to preferential stable adsorption site at the stepfoot and energy barrier at the upper step edge. This asymmetric profile is known as the EhrlichSchwoebel barrier ES [9]. Kinetically speaking, the ES barrier induces a stabilizing effect in the step train direction since the steps grow essentially by capture of atoms diffusing on the lower terraces, 
while incorporation of atoms from the upper terraces to step edges is prevented or drastically reduced. This step flow growth is at the origin of the ideal layer-by-layer homo-epitaxial growth. It can be accompanied by a morphological instability, called step meandering, along the step direction [10], for which the straight step moves into a smooth and periodic profile with a characteristic Bales-Zangwill wavelength.

In this letter, we use kinetic Monte Carlo (KMC) simulations to study the hetero-epitaxial growth of $\mathrm{Ag}$ deposited on vicinal Pt surfaces. Previous calculations led us to extract the ideal conditions to obtain perfect monatomic Ag wires [7]. Here, we investigate the upper-wire coverage regime. STM experiments [11] of Ag decoration of Pt steps showed that, above $150 \mathrm{~K}$, large and dense Ag-islands grow away from the substrate steps. Their morphology changed from dendritic to compact behavior when rising $T$. A closer examination of the distribution of the islands along the steps led us to suspect a quasi-periodic arrangement of the atoms. We show here that KMC simulations based on atomistic processes can reproduce this feature and that the wavelength along the step can be tailored by changing the growth conditions (temperature, coverage, flux) and the terrace widths.

Various theoretical attempts were proposed $[12,13]$ to study the formation of gratings. Unfortunately, some limiting hypotheses (irreversible aggregation, semi-circular island shape and isotropic diffusion) [14] prevented to investigate the local properties of growth depending on the interaction potentials with the surface and between the adatoms themselves.

Here, we consider a regularly stepped Pt surface which is formed by close packed (1 111$)$ terraces of width $w$ and separated by parallel steps of length $L$. The lattice parameter for $\operatorname{Pt}\left(\begin{array}{lll}1 & 1 & 1)\end{array}\right)$ is $a=2.77 \AA$ and periodic conditions are applied along the length $L$ assumed to be fixed and equal to 300 sites, i.e. $831 \AA$, while the terrace width $w$ is varied from 10 to 70 lattice sites, i.e. from 27 to $168 \AA$. The $\mathrm{Ag}$ atoms are randomly deposited as monomers on the stable sites corresponding to the equilibrium structure of the $\mathrm{Ag}$ monolayer, with a flux $F$ per site and time units. Within the conditions of cov- erage $\theta \leqslant 0.3 \mathrm{ML}$ (ML stands for monolayer) and temperature $(T \leqslant 300 \mathrm{~K})$, atom deposition above already formed islands $[15,16]$ and desorption from the surface [17] are unprobable events. The diffusion at the $\mathrm{Pt}$ surface is modelled by transition state theory and an Arrhenius law is used to describe the jumping process of an atom above a barrier $\Delta E_{k}$. This barrier depends on the holding potential through the surface corrugation and on the lateral interactions which account for the initial and final environment of the jumping atom defining a process $k$. Hence, 61 microscopic processes $k$ were listed for the calculation of the set of $\Delta E_{k}$ barrier values. All these processes are defined as the jumps of a peculiar adatom from its initial equilibrium well towards an adjacent one (in general the hollow sites of $\operatorname{Pt}\left(\begin{array}{lll}1 & 1 & 1\end{array}\right)$ surface), by considering the influence of the other adatoms which are in a nearest neighbor position of the jumping atom in its initial and final wells (see Ref. [7] for the detail of the processes). These barriers were thus calculated with semi-empirical potentials based on tight binding theory in the second moment approximation [7]. The use of such potentials is very useful for CPU time gain and their accuracy has been tested by comparing with STM data and Effective Medium Theory calculations [18]. As an example, the barrier $\Delta E$ is equal to $0.143 \mathrm{eV}$ for an isolated monomer (no nearest neighbor) diffusing on a perfect surface and arriving in an adjacent site without any neighbor. But this value of $\Delta E$ changes from 0.02 to $0.64 \mathrm{eV}$ depending on the number of neighbors around the jumping atom and on the step proximity. The diffusion coefficient $D_{k}$ connected to the $k$ th process is then given by $D_{k}=D_{0} \exp \left(-\Delta E_{k} / k T\right)$ where the prefactor $D_{0}$ takes the usual value $10^{12} a^{2}\left(\AA^{2} \mathrm{~s}^{-1}\right)$. The aggregation is reversible and can thus account for the transition from ramified to compact two-dimensional $\mathrm{Ag}$ islands on Pt.

Fig. 1 displays typical snapshots of kinetic Monte Carlo (KMC) simulations at constant flux $\left(F=10^{-3} \mathrm{ML} \mathrm{s}^{-1}\right)$ and coverage for two temperatures $T$ and three widths $w$. For an intermediate terrace width $(w=30 a)$ and at low temperature $(T=120 \mathrm{~K})$, a fractal-like step coating is obtained with the initial formation of one atomic chain along each step. When $T$ grows to $200 \mathrm{~K}$, the step 

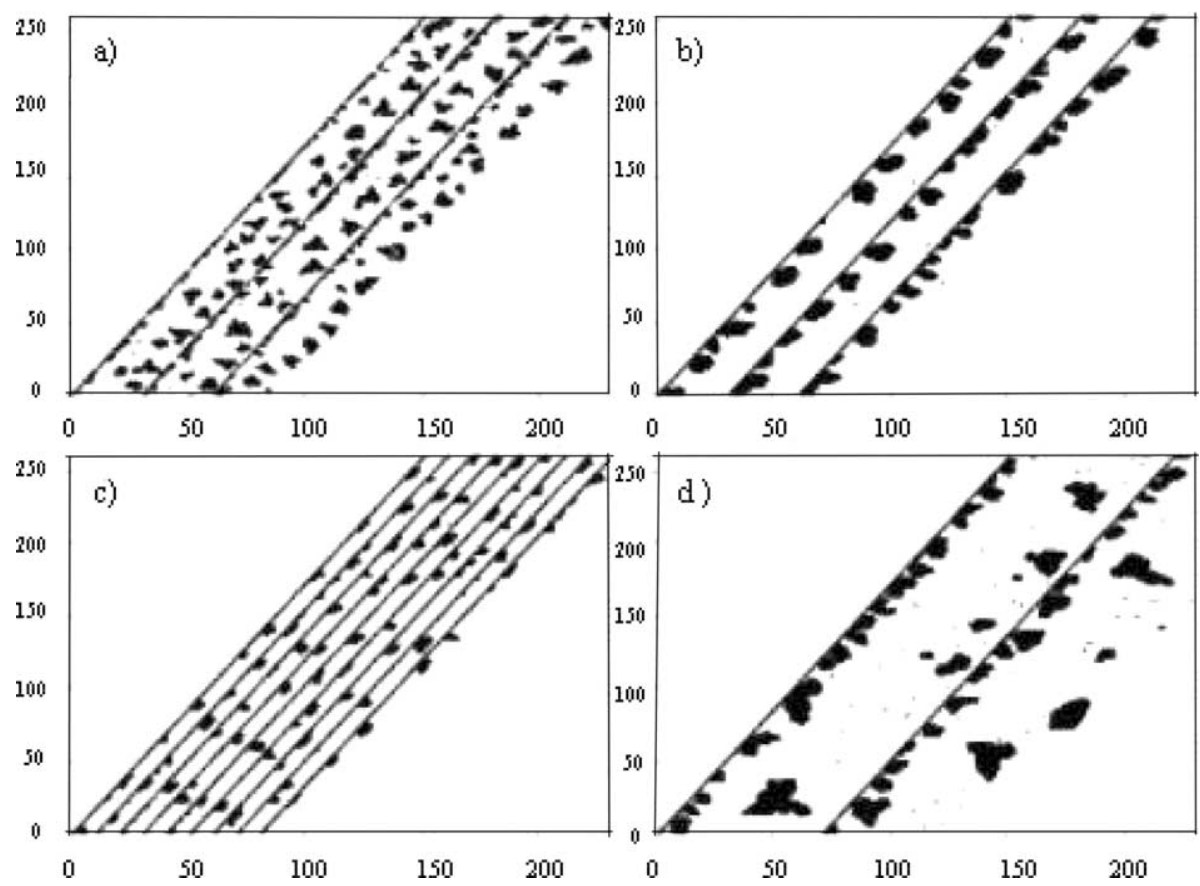

Fig. 1. Snapshots of $0.18 \mathrm{ML} \mathrm{Ag}$ adatoms deposited on a $(w \times L)$ Pt terrace at temperature $T$ with a deposition flux $F=10^{-3} \mathrm{ML} \mathrm{s}^{-1}$. The following values were used (in all case $L=300 a$ ): (a) $w=30 a, T=120 \mathrm{~K}$; (b) $w=30 a, T=200 \mathrm{~K}$; (c) $w=10 a, T=200 \mathrm{~K}$; (d) $w=70 a, T=200 \mathrm{~K}$. At small terrace width and sufficiently small temperature, quasi-perfect monatomic unidirectional chains are formed. Otherwise, a nearly regular pattern attached to these chains develops with an associated wavelength $\lambda$.

decoration is more pronounced and all the islands are attached to the step via the first chain. Compact islands clearly prevail over fractal ones, and only a very small number of terrace islands lying far apart from the steps are observed for the wider terraces.

In all the situations shown in Fig. 1, the striking feature is the nearly regular distance which characterizes the island separation, or equivalently, the existence of a typical wavelength $\lambda$ characteristic of this periodicity. At first glance, this periodicity is not obvious since the islands have not exactly the same size and shape. Fourier transform (FT) procedure should in principle be powerful to analyze such periodic systems. However, the island size dispersion can bring additional noise. To quantitatively prove this feature, we have performed a KMC simulation on a single step edge characterized by a terrace width of $w=10 a$ and a large length $L=2700 a$ (in order to have better statistics). Fig. 2 exhibits the FT signal obtained at $T=200 \mathrm{~K}$. The narrow and intense peak at $\lambda / a=31$ proves that this system is clearly periodic. For a set of identical steps (vicinal surfaces) CPU time drastically grows and it is thus necessary to decrease the step length. Statistics is then applied on a large number of smaller steps and we have preferred to calculate the average distance between the center of mass of the islands over a large number of snapshots, the FT approach giving more intricate signals. This periodicity appears to be in agreement with the features observed in a revisited examination of the original STM experiments [11]. Therefore, several questions arise, namely: (i) Why a first row of silver atoms is formed before islands take place and why periodically spaced islands then nucleate on vicinal surfaces? (ii) Can the periodicity of the islands be controlled by the external parameters used in MBE? (iii) Which conditions lead to a well-defined wavelength in a large temperature range?

In order to interpret this heterogeneous growth and especially to explain the first row and island 


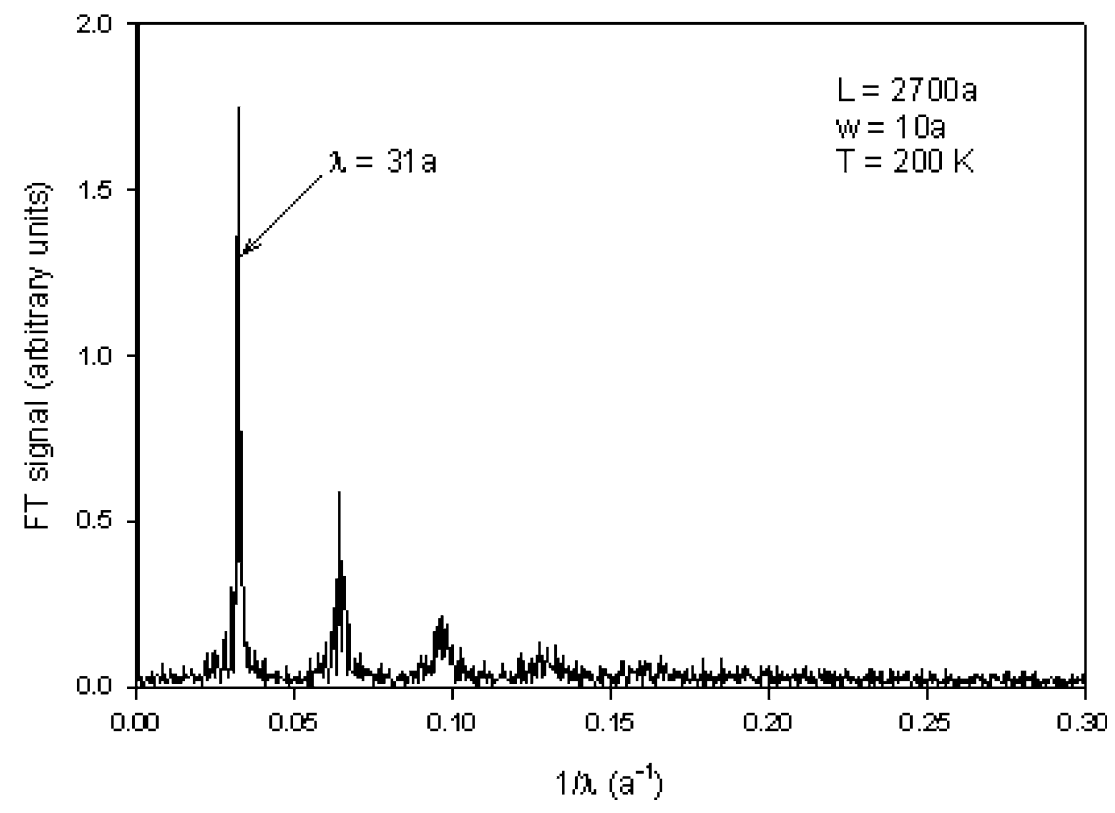

Fig. 2. Fourier transform of the inter-distance island distribution obtained from a KMC simulation of $0.18 \mathrm{ML}$ Ag adatoms deposited on a $(w \times L)$ Pt terrace at temperature $T=200 \mathrm{~K}$ with a deposition flux $F=10^{-3} \mathrm{ML} \mathrm{s}^{-1}$. Here, $w=10 a$ and $L=2700 a$, in order to get good statistics. The system is found to be periodic (4 harmonics are observed) with a characteristic wavelength $\lambda / a=31 \pm 2$.

formation, let us recall the competition between the nucleation regime and step flow growth of homogeneous misoriented surfaces discussed by Fuenzalida [19]. He showed that the condition under which step flow growth dominates is a function of a reduced growth parameter $g=$ $F w^{4} / D$ where $D$ represents the isotropic diffusion coefficient of monomers on the perfect surface. This parameter represents the competition between the diffusion time $\left(\sim D^{-1}\right)$ and the deposition time $\left(\sim F^{-1}\right)$. For $g$ values larger than unity occurring for large flux, low temperature and/or large terrace, the adatoms have not enough time to diffuse toward the steps and the nucleated islands which appear on the terraces prevent the step flow growth. In Fig. 1a obtained for $w=30 a, T=120$ $\mathrm{K}$ and Fig. 1d obtained for $w=70 a, T=200 \mathrm{~K}$, the values of $g$ are equal to 242 and 32, respectively, using the activation barrier of monomers on the terrace. This results in nucleated islands on the terraces. In the opposite situation (Fig. 1c), i.e. for $w=10 a, T=200 \mathrm{~K}, g$ becomes very small $(g=0.01)$. Step flow growth proceeds and atoms completely condensate at step foots. When $g$ reaches its critical value $(g=1.08)$ for $F=0.001$ $\mathrm{ML} \mathrm{s}^{-1}, w=30 a$ and $T=200 \mathrm{~K}$, only small islands effectively occur on the terraces at the end of deposition time (Fig. 1b), thus demonstrating the sensitivity of this parameter $g$ in the heterogeneous growth. However, it should be noted that Fuenzalida [19] used a mean field description for the steady state whereas our simulations start from the earlier stages of growth and span the transient regime.

These arguments can also be transposed to understand the completion of the Ag rows. To determine whether the $j$ th row is fully occupied or not, we calculate the ratio of the diffusion coefficients $D_{j+1} / D_{j}$ for a $\mathrm{Ag}$ atom in the $(j+1)$ th and $j$ th rows. This ratio is directly connected to the probability for this atom to detach from this row and move to the adjacent row ( Fig. 3). From the knowledge of the activation barrier $\Delta E_{1}=0.50 \mathrm{eV}$ and $\Delta E_{2}=0.27 \mathrm{eV}$, and assuming reasonably the same prefactor constant for the first and second row, the ratio $D_{2} / D_{1}$ equals to $6 \times 10^{5}$ at $200 \mathrm{~K}$. As a consequence, every adatom statistically spends much more time in the first row than in the second 


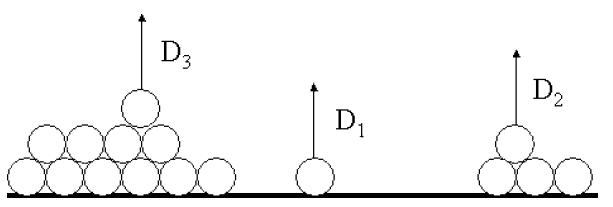

Fig. 3. Schematic view of the detachment processes responsible for the nucleation against completion of $\mathrm{Ag}$ wires at the Pt step. $D_{1}$ is the diffusion constant for a monomer trapped in the $\mathrm{Pt}$ step. $D_{2}$ and $D_{3}$ correspond to the detachment of an $\mathrm{Ag}$ atom from the second and third $\mathrm{Ag}$ row.

row. It can detach easily from the second row, but because of the step flow growth condition (small value of $g$ and step coating), it diffuses on the terrace up to reach an unoccupied first row site. When the first row of $\mathrm{Ag}$ is filled, the Schwoebel effect experienced by the second row Ag adatoms changes into a barrier which is characteristic of the $\mathrm{Ag}-\mathrm{Ag}$ instead of the $\mathrm{Ag}-\mathrm{Pt}$ interactions. The activation barrier $\Delta E_{3}=0.24 \mathrm{eV}$ is very close to the barrier $\Delta E_{2}=0.27 \mathrm{eV}$ and the value of the ratio $D_{3} / D_{2}$ becomes equal to 5 . Statistically, the time spent by any atom in the second or third rows is nearly the same and no additional information is able to discriminate the second and third step coating. As a result, the atoms belonging to these rows are similarly trapped in their sites and they cannot complete the second row. The growth mechanism proceeds via the nucleation of islands from this row. This peculiar behavior (coating of the Pt step and islands formation) corresponds to the $2 \mathrm{D}$ transposition of the 3D Stranski-Krastanov layer-plus island growth.

The quasi-periodicity observed in the $\mathrm{Ag} / \mathrm{Pt}$ system can thus be interpreted on the basis of nucleation arguments. The average distance between islands is closely related to the probability by which one monomer is equivalently captured by two adjacent islands. Observations of the homogeneous step flow growth of several copper vicinals by Maroutian et al. [20] led to the conclusion that these surfaces develop a step-meandering instability characterized by a specific wavelength. The wavelength selection can be done by tuning temperature and deposition flux. They also suggested that the observed wavelength is imposed by the nucleation regime. A one-dimensional nucleation length $L_{n}=\left(\frac{12 a D_{\text {step }}}{F_{w}}\right)^{1 / 4}$, along the step can be de- fined, where $D_{\text {step }}$ is an effective diffusion coefficient [21]. It is therefore tempting to assign the observed wavelength to this nucleation length since nucleation proceeds from the second row.

This is the aim of the second question to verify how this assumption can interpret the dependence of the wavelength $\lambda$ as a function of the parameters $(T, F, \theta)$ and terrace width $w$. For compact islands, $\lambda$ is simply calculated as the arithmetic average over 25 runs between the centers of gravity of adjacent islands. The island size distribution has also been taken into account through the calculation of the standard deviation $\sigma$. For fractal-like shapes, we determine the diffusion front attached to a source [22] that is represented by the step. Let $x_{\mathrm{f}}$ and $\sigma_{\mathrm{f}}$ be the mean distance and the width of the front, respectively, the wavelength $\lambda$ is thus calculated as the average gap between the relevant atoms located in the interval $x_{\mathrm{f}} \pm \sigma_{\mathrm{f}}$. Note that ramified islands occur when diffusion is hindered by temperature and also when the terrace size increases. They do not occur for narrower terraces ( $w=8 a$, such as the vicinal (997) Pt surface) which favor the formation of perfect wires along the steps [7].

For compact islands (i.e. $T \geqslant 200 \mathrm{~K}$ ), the wavelength $\lambda$ decreases from $\lambda / a=29 \pm 3$ to $20 \pm 1$ when $w$ increases from 10 to $70 a$, respectively (Fig. 4). This can be understood on basic arguments. The mean free path of the atoms along the step direction decreases when $w$ increases for a trivial reason connected to the increasing number of sites explored by the atoms on a wider terraces. Consequently, atoms adsorbed on step sites probe less sites and create more nucleation centers, resulting in a decrease of $\lambda$. This feature is corroborated by examination of the wavelength distribution, which narrows rapidly when $w$ increases. A linear regression applied to the curve $\ln (\lambda)$ vs $\ln (w)$ (inset in Fig. 4) gives the modified scaling law $\lambda \propto w^{-\gamma}$. The calculated values of $\gamma$ range between $1 / 4$ for $\theta \leqslant 0.12 \mathrm{ML}$ to $1 / 6$ for $\theta>0.24 \mathrm{ML}$. These scaling factors are commonly obtained from rate equations for the mean distance between islands in the nucleation $(\gamma=1 / 4)$ and coalescence $(\gamma=1 / 6)$ regimes on infinite terraces [23].

Fig. 5 shows that $\lambda$ displays two distinct regimes vs temperature. When $T \leqslant 220 \mathrm{~K}$, the growth is 


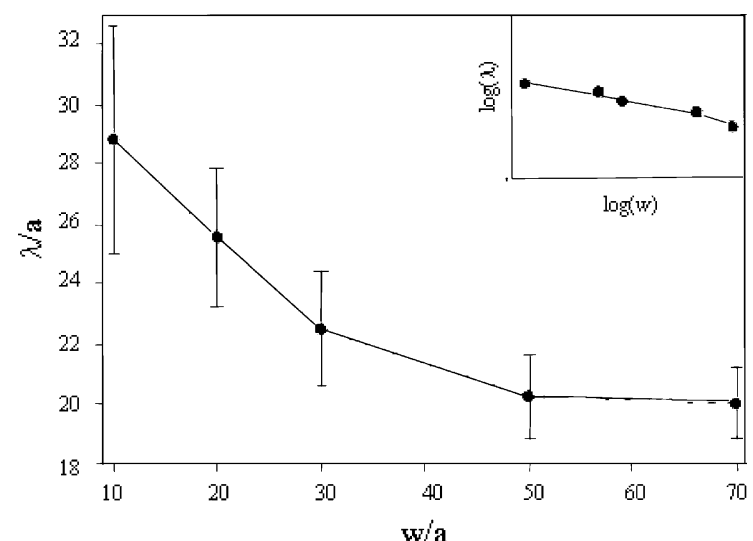

Fig. 4. Wavelength dependence vs the width $w$ of the Pt terraces in reduced unit $a . \theta=0.24 \mathrm{ML}, T=200 \mathrm{~K}, F=10^{-3}$ $\mathrm{ML} \mathrm{s}^{-1}$ and $L=300 \mathrm{a}$. The error bars shows how the gap between the islands attached to the steps is distributed. Narrow distributions are obtained when $w$ increases, leading to wellcharacterized gratings. The inset shows the linear regression $\log (\lambda)$ vs $\log (w)$ with a critical exponent equal to $-1 / 4$.

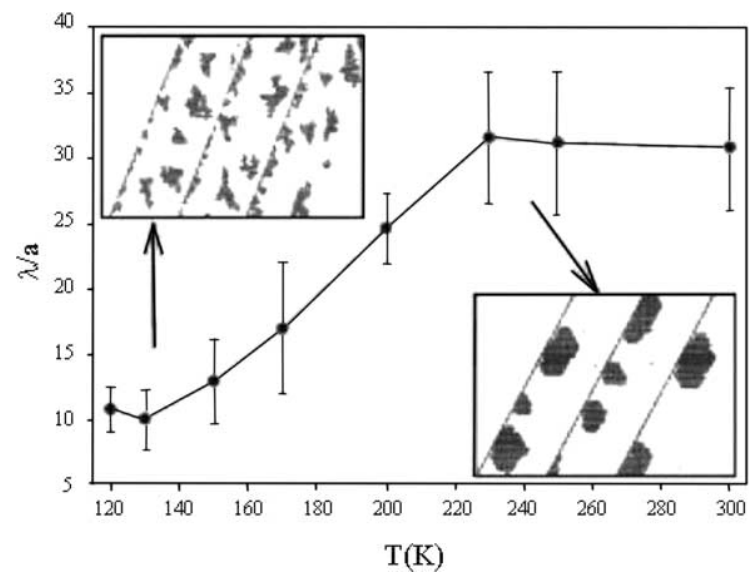

Fig. 5. Wavelength dependence vs temperature $T$ for a $(w \times L)=(30 a \times 300 a)$ Pt terrace, $\theta=0.27 \mathrm{ML}$ and $F=10^{-3}$ $\mathrm{ML} \mathrm{s}^{-1}$, showing two transitions: Fractal to compact shape for the islands and kinetic to thermodynamic regime for $\lambda$.

governed by kinetic effects since diffusion is hindered. The periodicity increases strongly with $T$ due to the mean square displacement of the atoms. For $T \geqslant 220 \mathrm{~K}$, the thermodynamic regime dominates and the wavelength saturates at $\lambda / a \simeq$ $30 \pm 4$. This latter value depends significantly on $w$, and more slightly on the coverage. From a structural point of view, we have observed a transition from fractal to compact islands between 150 and $170 \mathrm{~K}$. In this temperature range, a strong decrease of the fractal dimension is calculated, from the standard value 1.7 obtained at low temperature (below $150 \mathrm{~K}$ ) down to unity at $170 \mathrm{~K}$. In Fig. 5, we have shown these typical behaviors in the two insets.

Although the behaviors of $\lambda$ vs coverage $\theta$ and deposition flux $F$ are not shown here, let us mention that we find the same scaling factors for $w$ at different coverage and fluxes. The nucleation arguments used to interpret the homogeneous step flow growth of $\mathrm{Cu}$ vicinals [20] seem to qualitatively reproduce the observed $\lambda$ scaling law. We thus propose the same law for the heterogeneous system, i.e. $\lambda=\left(\frac{12 a D_{\text {step }}}{F w}\right)^{\gamma}$ even if in our case the set of diffusion processes $D_{k}$ cannot be simply represented by a single diffusion coefficient $D_{\text {step }}$. Nevertheless, this law leads to realistic values for $\lambda$ when we take an average value $\frac{\Delta E_{2}+\Delta E_{3}}{2}=0.25 \mathrm{eV}$ for the diffusion barrier occurring in $D_{\text {step }}$. For instance, at low coverage $(\gamma=1 / 4), F=10^{-3}$ $\mathrm{ML} \mathrm{s}^{-1}, w=10 a$, and $T=200 \mathrm{~K}$, the calculated value $\lambda=24 a$ is close to the $\mathrm{KMC}$ result $\lambda=28 a$. In the same conditions, $\lambda$ is found to be equal to $19 a$ and $15 a$ for $w=30 a$ and $w=70 a$, respectively. These latter values are not in contradiction with those obtained with the simulations $(\lambda=22 a$ and $19 a$, respectively). An important feature resulting from this law is that it could explain why $\lambda$ remains constant at $T>220 \mathrm{~K}$ since all the diffusion processes become equivalent when the system reaches its thermodynamic regime.

Finally, the influence of defects (kinks) along the steps on the behavior of the wavelength $\lambda$ vs $w$ and $T$ has been simulated by periodically distributing along the steps irreversible nucleation centers with a period $L_{0}$. The wavelength $\lambda$ displays two regimes. When $L_{0}<\lambda, \lambda$ follows a linear law with $L_{0}$ which is independent on $w$. For $L_{0}>\lambda$, the behavior obtained for perfect steps, $\lambda \propto w^{-\gamma}$ is recovered whatever the terrace width $w$ and the temperature. Note here that Pt steps, like terraces, have been considered as ideally structured. This could not be the case due to step relaxation, since it is known that the edge Pt atoms tend to slightly move inside the bulk [24]. This is however a very 
small effect which should be partly counterbalanced by the presence of $\mathrm{Ag}$ atoms at the steps. Another cause of defects could be due to $\mathrm{Pt} / \mathrm{Ag}$ exchange but it seems excluded at this temperature [6].

To summarize, vicinal surfaces are convenient templates to fabricate one-dimensional wires and gratings with controlled shape and periodicity at the nanometer scale. Nucleation length is at the origin of these peculiar features. When the thermodynamic regime prevails, the wavelength connected to the grating periodicity depends mainly on the terrace width. The Ag coating step of $\mathrm{Pt}$ vicinals has been chosen as an example to obtain simultaneously monatomic wires and grating attached to them, but the present conclusions could be extended in a straightforward way to other systems.

\section{References}

[1] M.G. Lagally (Ed.), Kinetics of Ordering and Growth at Surfaces, Plenum, New-York, 1990.

[2] H. Röder, E. Hahn, H. Brune, J.P. Bucher, K. Kern, Nature 366 (1993) 141.

[3] H. Brune, Surf. Sci. Rep. 31 (1993) 121.

[4] J.A. Venables (Ed.), Introduction to Surface and Thin Film Processes, Cambridge University Press, 2000.

[5] T. Zambelli, J. Wintterlin, J. Trost, G. Ertl, Science 273 (1996) 1688.
[6] P. Gambardella, M. Blanc, H. Brune, K. Kuhnke, K. Kern, Phys. Rev. B 61 (2000) 2254.

[7] F. Picaud, C. Ramseyer, C. Girardet, P. Jensen, Phys. Rev. B 61 (2000) 16154

[8] H. Brune, M. Giovannini, K. Bromann, K. Kern, Nature 394 (1998) 451

[9] R.L. Schwoebel, J. Appl. Phys. 40 (1969) 614.

[10] G.S. Bales, A. Zangwill, Phys. Rev. B 41 (1990) 5500.

[11] H. Röder, H. Brune, J.P. Bucher, K. Kern, Surf. Sci. 298 (1993) 121.

[12] T. Vicsek, Phys. Rev. Lett. 53 (1984) 2281.

[13] C.D. Pownall, P.A. Mulheran, Phys. Rev. B 60 (1999) 9037.

[14] A.K. Myers-Beaghton, D. Vvedenski, Phys. Rev. B 42 (1990) 5544

[15] P. Zeppenfeld, J. Goerge, M. Büchel, R. David, G. Comsa, Surf. Sci. Lett. 318 (1994) L1187.

[16] C. Mottet, R. Ferrando, F. Hontinfinde, A.C. Levi, Surf. Sci. 417 (1998) 220.

[17] P. Jensen, H. Larralde, A. Pimpinelli, Phys. Rev. B 55 (1997) 2556.

[18] H. Brune, K. Bromann, H. Röder, K. Kern, Phys. Rev. B 52 (1995) R14380.

[19] V. Fuenzalida, Phys. Rev. B 44 (1991) 10835.

[20] T. Maroutian, L. Douillard, H.J. Ernst, Phys. Rev. Lett. 83 (1999) 4353;

T. Maroutian, L. Douillard, H.J. Ernst, Phys. Rev. B 64 (2001) 165401.

[21] P. Politi, J. Villain, Phys. Rev. B 54 (1996) 5514; P. Politi, J. Phys. I France 7 (1997) 797.

[22] B. Sapoval, M. Rosso, J.F. Gouyet, J. Phys. Lett. 46 (1985) L149.

[23] G.S. Bales, D.C. Chzran, Phys. Rev. B 50 (1994) 6057.

[24] P. Steadman, K.F. Peters, H. Isern, S. Ferrer, Phys. Rev. B 64 (2001) 125418. 